\title{
ENGENHARIA BASEADA EM CONHECIMENTO APLICADA AO CONCEITO DFMA: ANÁLISE BIBLIOMÉTRICA E SISTÊMICA
}

Otávio Gobbo Junior (otaviojunior@alunos.utfpr.edu.br) - PPGEM, Universidade Tecnológica Federal do Paraná

Lucas Barboza Zattar Paganin (paganin@alunos.utfpr.edu.br) - PPGEM, Universidade Tecnológica Federal do Paraná

Milton Borsato (borsato@utfpr.edu.br) - PPGEM, Universidade Tecnológica Federal do Paraná

\section{RESUMO}

No Processo de Desenvolvimento de Produto (PDP) a gestão do conhecimento é essencial para evitar retrabalhos futuros. Uma abordagem para gerir o conhecimento necessário ao desenvolvimento do produto é denominada Engenharia Baseada em Conhecimento (Knowledge-based Engineering - KBE). Soluções de KBE permitem automatizar ou personalizar uma atividade de projeto utilizando, por exemplo, um sistema especialista. Uma ferramenta com esta característica poderia apoiar equipes de projeto para aplicar o conceito de Projeto para Fabricação e Montagem (Design for Manufacturing and Assembly - DFMA), tradicionalmente dependente do conhecimento do projetista e da comunicação com outros departamentos ou fornecedores. Nessa perspectiva, o presente artigo propõe a construção de uma base de publicações relacionadas à aplicação de KBE no DFMA, com o objetivo de identificar as soluções encontradas pelos autores e constatar as tendências e oportunidades de pesquisa. Para tanto, foi aplicado o processo ProKnow-C (Knowledge Development Process - Constructivist) para seleção e análise dos artigos científicos em etapas, alcançando-se um portfólio bibliográfico composto de 22 artigos. As análises desses artigos respaldaram o produto final deste trabalho, com a identificação dos problemas de pesquisa, as soluções produzidas pelos estudos e as oportunidades de pesquisa proporcionadas, servindo de amparo para o desenvolvimento de um sistema especialista com tal finalidade.

Palavras-chave: KBE; Design for Manufacturing and Assembly; DFMA; Análise bibliométrica; Análise Sistêmica

Área: Gestão do conhecimento

\section{INTRODUÇÃO}

A disponibilidade do conhecimento necessário para o desenvolvimento de um novo produto é essencial para o sucesso de um projeto. Segundo Rozenfeld et al. (2006), durante o Processo de Desenvolvimento de Produtos (PDP) deve-se armazenar informações e experiências constantemente. Os mesmos autores completam que o acúmulo do conhecimento e o uso de ferramentas de TI apropriadas permitirão a reutilização dessas informações, evitando a ocorrência de erros já cometidos. Uma abordagem para gerir o conhecimento necessário ao desenvolvimento do produto é denominada Engenharia Baseada em Conhecimento (Knowledge-based Engineering - KBE).

A Engenharia Baseada em Conhecimento pode ser entendida como soluções que apoiam atividades de engenharia ao realizar a coleta, estruturação e armazenamento de um 
conhecimento, ficando esse capital intelectual disponível para reutilização e regeneração (CANDIA et al., 2016). Um exemplo de solução de KBE são os sistemas especialistas.

De acordo com Ipek et al. (2013), um sistema especialista é uma ferramenta computacional interativa que utiliza fatos e heurísticas para apontar soluções para problemas difíceis, baseando-se no conhecimento obtido de um especialista. No PDP, um sistema especialista poderia automatizar ou personalizar uma atividade de projeto até então dependente do conhecimento de um profissional experiente, ou de informações encontradas em livros, normas, dentre outros.

Um exemplo de atividade onde a abordagem KBE utiliza um sistema especialista para auxiliar na concepção de um artefato é a orientação do projeto para a fabricação, dentro do conceito denominado Projeto para Manufatura e Montagem (Design for Manufacturing and Assembly - DFMA), ou simplesmente para a manufatura (Design for Manufacturing - DFM) ou para a montagem (Design for Assembly - DFA). No trabalho de Krogstiea e Anderson (2012), percebeu-se que erros de projetos que impactavam negativamente na fabricação eram recorrentes, e que o conhecimento adquirido pelos profissionais da produção poderia ser mais bem aproveitado no projeto.

Nesse cenário, o presente trabalho apresenta uma revisão da literatura, identificando as soluções propostas em estudos publicados nos últimos cinco anos, com o objetivo de compreender o estado da arte e enxergar oportunidades de pesquisa existentes para a aplicação da KBE dentro do conceito de DFMA.

Para atingir tal objetivo, utilizou-se o processo ProKnow-C, que colabora na fundamentação de uma pesquisa científica. Esse processo consiste basicamente na composição de um portfólio bibliográfico, análise bibliométrica e análise sistêmica acerca do tema pesquisado (ENSSLIN, 2010).

Este artigo é composto de 6 seções. A primeira seção é composta pela introdução. Já na seção 2 são apresentados os aspectos metodológicos. Nas seções 3,4 e 5 será apresentado o desenvolvimento da pesquisa, com a seleção do portfólio bibliográfico, análise bibliométrica e sistêmica respectivamente. Por fim, a seção 6 trará as considerações finais.

\section{ASPECTOS METODOLÓGICOS}

O objetivo desta seção será a apresentação dos passos percorridos para se chegar ao estado da arte sobre o tema em estudo. $\mathrm{O}$ procedimento metodológico adotado foi o denominado Knowledge Development Process-Constructivist (ProKnowC), que colabora para que os pesquisadores encontrem os conteúdos mais expressivos dentro de uma extensa quantidade de informações na literatura (ENSSLIN et al., 2010).

O processo ProKnow-C é dividido em quatro etapas (Figura 1). Segundo Ensslin et al. (2010), a primeira etapa, construção do portfólio de artigos, consiste na seleção de um banco de artigos bruto e na filtragem destes artigos. A segunda etapa é a análise bibliométrica do portfólio, na qual ocorre uma análise de quais são os periódicos e as palavras-chave mais recorrentes, além do número de vezes que cada artigo foi citado na literatura com o propósito de se identificar as publicações mais relevantes para o tema de pesquisa. No terceiro passo, análise sistêmica, é realizada a leitura integral do portfólio resultante, a fim de identificar os problemas de pesquisa, as soluções propostas pelos autores e as oportunidades de pesquisa observadas. Por fim, a última etapa do ProKnow-C é a definição da pergunta e dos objetivos de pesquisa. Entretanto, este estudo limitou-se nas três primeiras etapas do processo, não adentrando no campo de um trabalho futuro. 
Figura 1 - Etapas do processo Proknow-C. Fonte: Adaptado de Ensslin et al. (2010).

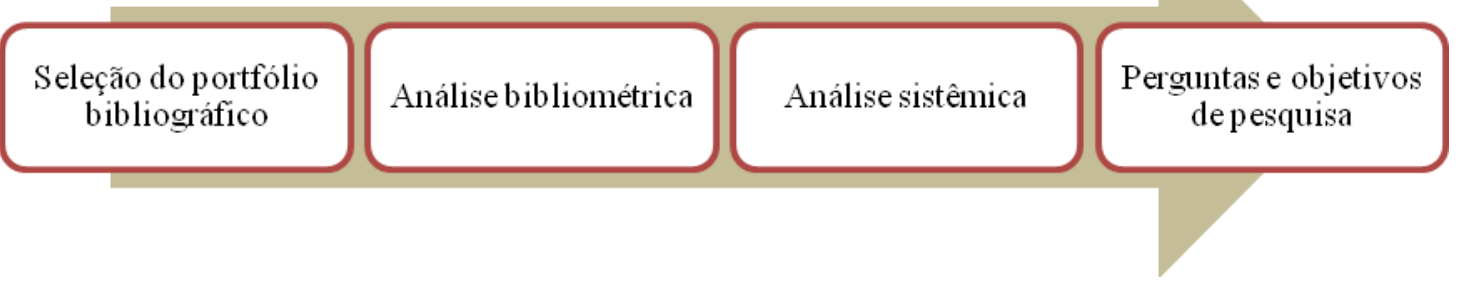

A pesquisa por artigos foi limitada nos últimos cinco anos, mais precisamente de janeiro de 2012 a março de 2017, e ocorreu em dez bases de dados escolhidas, que são elas: ProQuest, Engineering Village, Scopus, Web of Science, Wiley, Emerald, Springer, Science Direct, EBSCO e IEEE. Para gerir o portfólio de artigos foi utilizado o software EndNote, e a estruturação das análises bibliográfica e sistêmica foi realizada no software Microsoft Excel.

\section{SELEÇÃO DO PORTFÓLIO BIBLIOGRÁFICO}

\subsection{Seleção da base de artigos bruta}

Para a construção do portfólio bibliográfico foram definidos primeiramente os eixos de pesquisa, que representam as áreas envolvidas com o tema explorado. Como o objetivo deste trabalho é analisar a engenharia baseada em conhecimento (KBE) aplicada ao projeto para manufatura e montagem (DFMA), observa-se que ambos, KBE e DFMA, são áreas de estudo distintas, portanto foram os dois primeiros eixos escolhidos. Ainda, pelo fato de este cenário estar inserido no Processo de Desenvolvimento de Produto (PDP), adotou-se este como terceiro eixo.

$\mathrm{Na}$ sequência, para cada eixo de pesquisa foram definidas algumas palavras-chave. Essas palavras foram adotadas após uma breve consulta na literatura para certificar os termos mais utilizados e seus possíveis sinônimos. Todas as palavras-chave adotadas são apresentadas na Tabela 1, divididas pelos três eixos de pesquisa.

A próxima etapa foi a busca por artigos nas bases de dados escolhidas, com o período limitado aos últimos cinco anos. Os resultados levaram ao total de 1.217 publicações, que compuseram a base bruta de artigos.

Tabela 1 - Eixos de pesquisa e palavras-chave. Fonte: Autoria própria.

\begin{tabular}{|c|c|}
\hline Eixos de Pesquisa & Palavras-chave \\
\hline $\begin{array}{c}\text { Projeto para manufatura e } \\
\text { montagem (DFMA) }\end{array}$ & $\begin{array}{c}\text { Design for Manufacturing and Assembly; DFMA; Design for } \\
\text { Manufacturing; DFM; Design for assembly; DFA;DFX }\end{array}$ \\
\hline $\begin{array}{c}\text { Engenharia baseada no } \\
\text { conhecimento (KBE) }\end{array}$ & $\begin{array}{c}\text { Knowledge-based engineering; KBE; knowledge management; } \\
\text { Knowledge-based systems; Expert systems; Intelligent systems; } \\
\text { Artificial intelligence; Ontology }\end{array}$ \\
\hline $\begin{array}{c}\text { Processo de desenvolvimento de } \\
\text { produto (PDP) }\end{array}$ & $\begin{array}{c}\text { Product development; Product development process; PDP; New } \\
\text { product development; NPD; Concurrent engineering }\end{array}$ \\
\hline
\end{tabular}




\subsection{Filtragem do banco de artigos}

A partir da amostra obtida pela etapa de seleção do portfólio bibliográfico, as publicações foram exportadas para o software EndNote para facilitar o gerenciamento dos artigos. Primeiramente, foi realizada uma eliminação dos artigos duplicados, reduzindo-se a base de 1.217 para 863 publicações.

$\mathrm{Na}$ sequência, foi realizada uma leitura dos títulos, admitindo para a próxima fase apenas artigos cujo título apontasse uma estreita relação com o objeto de pesquisa. Restaram-se apenas 31 artigos para análise posterior. Dessa maneira, utilizando-se do Google Scholar, foi possível identificar o número de vezes que cada publicação havia sido citada por outros autores com o intuito de se verificar o reconhecimento científico de cada artigo. Após esta apuração, foi estabelecida a eliminação de artigos que nunca haviam sido citados, com exceção dos títulos do ano de 2016, os quais permaneceram na amostra devido ao pouco tempo para serem citados em novos trabalhos. Desta forma, 25 artigos seguiram para a etapa de análise seguinte.

A última etapa para seleção e triagem dos artigos consistiu na leitura dos resumos das 25 publicações. Assim, concluiu-se que 3 artigos poderiam ser eliminados pois não eram relevantes ao objetivo da pesquisa, e desta forma chegou-se a um número de 22 artigos reconhecidos cientificamente e alinhados com o objetivo da pesquisa, os quais compuseram o portfólio bibliográfico.

\section{ANÁLISE BIBLIOMÉTRICA}

A análise bibliométrica assessora a interpretação e a avaliação do portfólio bibliográfico, por meio de uma análise quantitativa dos artigos pelo ano de publicação, pelo reconhecimento científico através do número de citações obtidas, e por uma análise das palavras-chave e periódicos mais recorrentes tocantes ao objeto do estudo (ENSSLIN, 2010).

Observa-se que houve um crescimento no número de publicações enquadradas com este estudo, alcançando o ponto máximo em 2014 com 7 publicações. Contudo, em 2015 e 2016 houve uma significativa redução, o que poderia alertar uma possível obsolescência do tema. Uma hipótese admissível para o fenômeno da Figura 2, é que as pesquisas com DFMA vêm decrescendo, enquanto que o interesse em KBE está no sentido oposto, servindo ainda de motivação para este estudo.

A próxima avaliação se referiu ao número de citações obtidas por cada artigo, retratado na Figura 3. Destaca-se o trabalho de Ipek et. al. (2013) com 39 citações.

Figura 2 - Quantidade de artigos pelo ano de publicação. Fonte: Autoria própria.

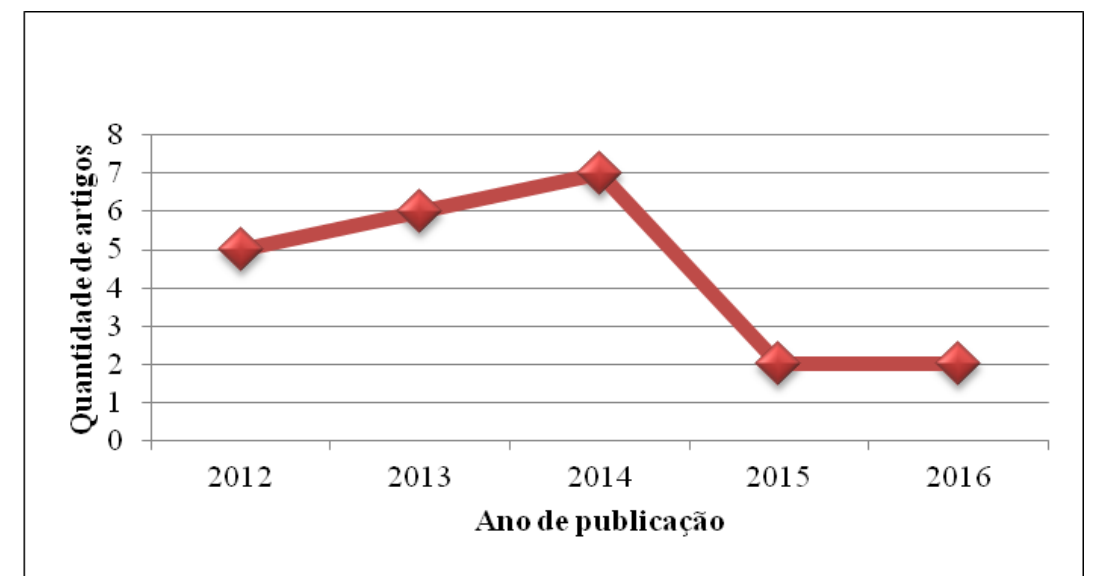


Figura 3 - Reconhecimento científico dos artigos pelo número de citações. Fonte: Autoria própria.

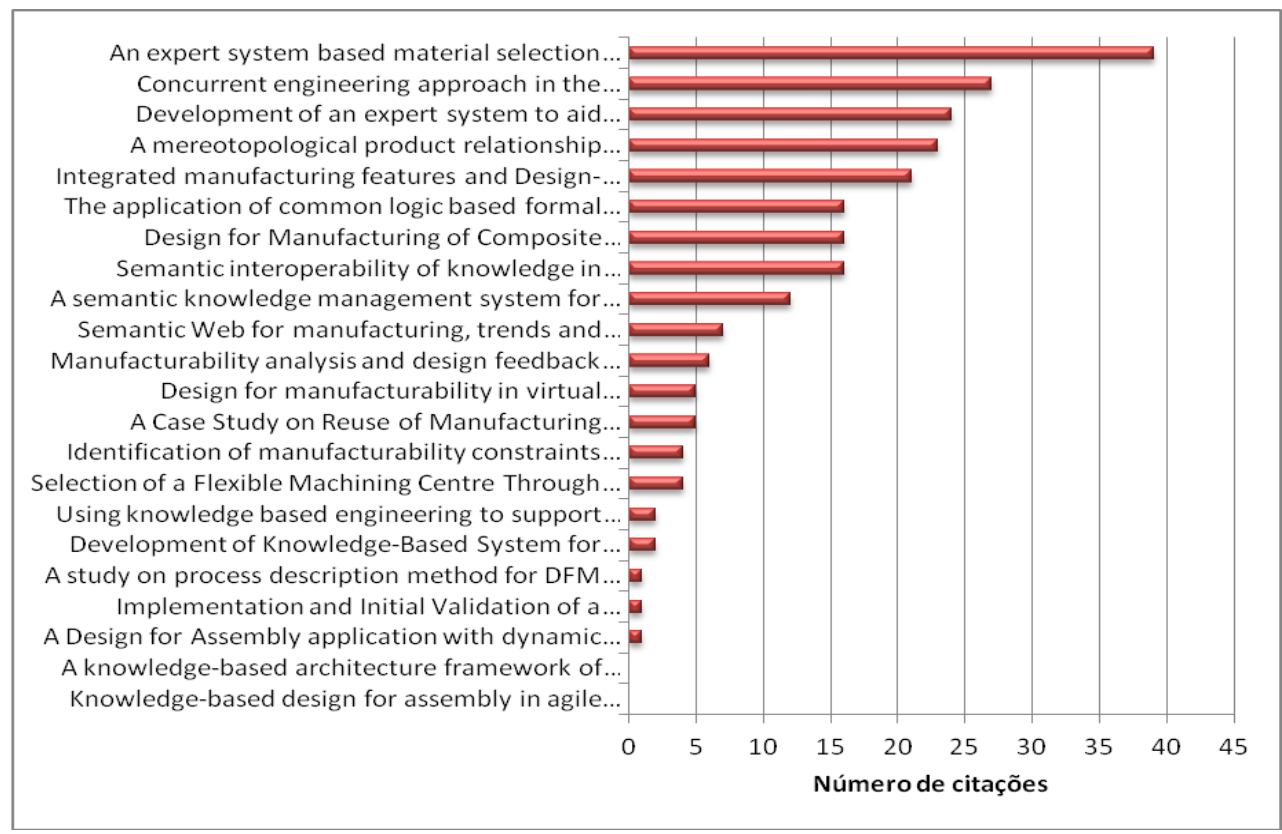

Outra apreciação foi quanto à aderência das palavras-chave, com a observação do número de ocorrências de cada uma no portfólio. Para isso, uniram-se as palavras que poderiam ser consideradas sinônimas ou muito semelhantes. Observa-se que as palavras mais recorrentes se referem à ontologia e KBE, ambas com sete ocorrências, seguidas por DFM e palavras que aludem à montagem conforme observado na Figura 4.

Para finalizar a análise bibliométrica, os periódicos ou conferências mais recorrentes foram levantados. Os que se destacaram com duas ocorrências foram: Advanced Engineering Informatics, CIRP Design Conference, Computers \& Industrial Engineering, IDETC/CIE ASME, Materials \& Design e Procedia CIRP. Essa análise é interessante para verificar onde são publicados mais artigos relacionados ao tema pesquisado.

Figura 4 - Aderência das palavras-chave. Fonte: Autoria própria.

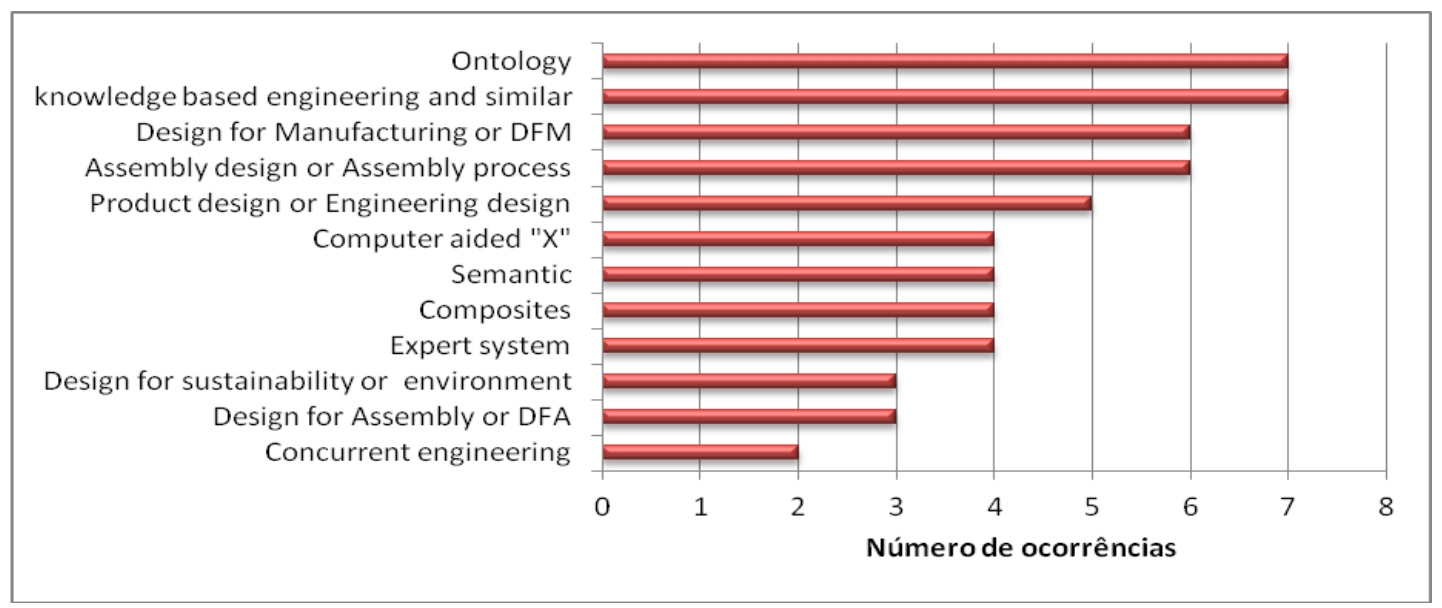

Terminada as análises quantitativas, iniciou-se a análise sistêmica do portfólio bibliográfico, a qual será apresentada na próxima seção. 


\section{ANÁLISE SISTÊMICA}

De acordo com Ensslin et al. (2010), a análise sistêmica é utilizada para explorar uma amostra representativa de um determinado assunto, visando identificar as oportunidades de pesquisa sobre o objeto em estudo. Após a leitura integral dos artigos realizando uma análise crítica, uma planilha foi elaborada contendo as seguintes informações extraídas de cada trabalho: objetivo, metodologia, principais resultados, recomendações futuras e oportunidades de pesquisa encontradas. A partir desses resultados, identificaram-se os problemas encontrados pelos autores, as soluções propostas e as oportunidades de pesquisa, apresentados a seguir de forma agrupada.

\subsection{Principais problemas de pesquisa}

\subsubsection{Modelos incompletos}

Diversos trabalhos abordaram a dificuldade para gerir ou armazenar o conhecimento simultaneamente com as informações geométricas no sistema CAD. Demoly, Matsokis e Kiritsis (2012) abordaram a necessidade de informações relativas à montagem. Já Hoque et al. (2013) questionaram a impossibilidade de agregar ao CAD o modo de fabricação da peça, quais ferramentas devem ser utilizadas, dentre outras características de fabricação. O estudo de Abdul-Ghafour et al. (2014) apontou que a falta de informações sobre como o modelo fora construído prejudica a interoperabilidade entre sistemas. Luetzenberger, Klein e Thoben (2013) trouxeram a necessidade de integração entre uma ontologia e o sistema CAD. Os trabalhos de Jakubowski e Peterka (2014) e Zhou e Saitou (2014) questionaram que dentro do ambiente CAD há liberdade para criar qualquer geometria sem se importar sobre como a peça será fabricada, prejudicando a manufaturabilidade. Zhou e Saitou (2014) apontaram como exemplo a dificuldade de fabricação dos cantos em peças estampadas.

\subsubsection{Representação do conhecimento da fabricação ou montagem}

Conforme demandado por Ramos (2015), Krogstiea e Anderson (2012), Iwaya, Rosso Junior e Hounsell (2013), Hiekata e Yamato (2014), Kretschmer et al. (2016) e Wahidin et al. (2016), há necessidade de representar melhor os dados de fabricação e montagem para que estes sejam aproveitados futuramente durante novos projetos. Sem uma ferramenta de auxílio, os registros de casos anteriores ficam a mercê da lembrança dos profissionais, e muitas vezes desperdiçados. Krogstiea e Anderson (2012) identificaram problemas semelhantes de comunicação em três empresas. Eles observaram a predominância da comunicação face a face, a pouca presença dos projetistas na produção, a distância entre os departamentos e a dificuldade de armazenar conhecimento para aproveitamento posterior.

\subsubsection{Problemas específicos na fabricação}

Os trabalhos de Sapuan e Mansor (2014), Anderson et al. (2014) e Premkumar et al. (2014) abordaram questões de fabricação envolvendo componentes de materiais compósitos. Devido às peculiaridades de fabricação, a manufatura de materiais compósitos predominou entre os trabalhos exclusivos para um único processo de fabricação. Para Premkumar et al. (2014), somou-se ainda a necessidade de integração entre as diversas ferramentas envolvidas para seleção de material, e ferramentas CAD e CAE. Já Zhou e Saitou (2014) buscaram solucionar problemas recorrentes na estampagem devido às características geométricas do produto. Jakubowski e Peterka (2014) apresentaram um caso no qual era necessário utilizar três ferramentas de usinagem para obedecer a um raio que não era importante, e se caso ele fosse alterado, apenas duas etapas com duas ferramentas eram necessárias. 


\subsection{Objetivos e recursos propostos}

\subsubsection{Sistema especialista baseado em regras}

A solução do problema por meio de um sistema especialista baseado em regras, e sem ontologia, foi adotada por Ipek et al. (2013), Chowdary e Muthineni (2012), Wahidin et al. (2016), Hernandez et al. (2012) e Iwaya, Rosso Junior e Hounsell (2013). Ipek et al. (2013) e Chowdary e Muthineni (2012) criaram um sistema baseado em regras IF-THEN, sendo que os primeiros autores o utilizaram para obter a resposta do material mais adequado e os outros para obter a máquina mais adequada para fabricação. Chowdary e Muthineni (2012) utilizaram a linguagem $\mathrm{C}$, um banco de dados e uma interface de fácil operação. Outros trabalhos, como de Hernandez et al. (2012), Iwaya, Rosso Junior e Hounsell (2013) e Kretschmer et al. (2016) buscavam no sistema um conceito como resposta, e não um conhecimento explícito, como um valor mensurável ou descrito, e criaram para isso um sistema baseado em casos semelhantes. Wahidin et al. (2016) propuseram um sistema especialista baseado em regras para planejar a montagem denominado DAEx, e para isso adquiriram conhecimento dos especialistas por entrevistas, assim como Anderson et al. (2014) e Krogstiea e Anderson (2012), e classificaram as várias concepções e técnicas de montagem.

\subsubsection{Soluções com ontologia}

Ontologias computacionais são capazes de modelar formalmente o conhecimento, podendo ser útil para determinada finalidade. Os trabalhos de Demoly, Matsokis e Kiritsis (2012), Abdul-Ghafour et al. (2014), Premkumar et al. (2014), Ramos (2015) e Hiekata e Yamato (2014) trouxeram essa ferramenta como solução. Tratando de DFA, Demoly, Matsokis e Kiritsis (2012) criaram uma sequência de montagem com um diagrama de Gantt e ontologia utilizando as linguagens OWL-DL e SWRL. As mesmas linguagens foram usadas por AbdulGhafour et al. (2014) para elaborar uma ontologia com interoperabilidade entre sistemas $\mathrm{CAD}$, de modo que carregue dados de modelagem do objeto além dos geométricos. Premkumar et al. (2014) recorreram a ontologia para integrar dados de materiais e softwares CAD e CAE. Hiekata e Yamato (2014) empregaram uma ontologia pela linguagem RDF e a API Java, Jena, para gerar um fluxo dos processos de fabricação. Alguns trabalhos, como de Demoly, Matsokis e Kiritsis (2012), Abdul-Ghafour et al. (2014) e Premkumar et al. (2014) citaram a utilização do software Protégé, um editor de ontologia e framework open-source para a construção de sistemas especialistas.

\subsubsection{Soluções integradas}

Premkumar et al. (2014) desenvolveram um sistema chamado SLACK, que integra dados de materiais (base de dados OptiData), de CAD (CREO), de FEA (Ansys) e de um software específico para compósitos (FiberSIM). A aquisição de dados do Ansys e FiberSIM se deu por arquivos XML e dos materiais por uma planilha do Excel. Luetzenberger, Klein e Thoben (2013) elaboraram uma ontologia chamada de Pacote de Aquisição de Conhecimento (KAW) ligando o CATIA com uma ontologia, conseguindo assim reunir toda a estrutura do produto e oferecer uma saída em XML. Já o trabalho realizado por Hoque et al. (2013) trouxe uma abordagem diferente para modelar o produto no software CAD, no qual desenvolveram um suplemento ao Creo Elements/Pro pela ferramenta ToolKit, que partindo da geometria da matéria-prima, permite desenhar na ordem das operações e com dimensões que são atendidas pelas ferramentas utilizadas para a fabricação. 


\subsection{Oportunidades de pesquisa identificadas}

\subsubsection{Aquisição do conhecimento}

Conforme observado nos artigos do portfólio, deve-se haver uma atenção especial para adquirir um conhecimento tácito, que ao contrário do explícito, não é definido por números e palavras. Dentre as formas de aquisição utilizadas nos estudos, destacaram-se a abordagem de casos semelhantes como realizada por Iwaya, Rosso Junior e Hounsell (2013) e Kretschmer et al. (2016), e a coleta de entrevistas de especialistas, conforme abordado nos trabalhos de Anderson et al. (2014), Krogstiea e Anderson (2012) e Wahidin et al. (2016). Embora o emprego de casos semelhantes tenha sido para DFA, seria oportuno também fazer proveito para uma aplicação de DFM.

\subsubsection{Utilização de ontologia}

Ramos (2015) sugeriu mais estudos para representação do conhecimento da fabricação por meio da ontologia, considerando a complexidade e heterogeneidade deste domínio. Luetzenberger, Klein e Thoben (2013) sugeriram um maior gerenciamento de ontologias dentro do ambiente CAD. De maneira geral, observa-se a capacidade das ontologias em explicitar conceitos, sendo uma ferramenta de grande potencial para o desenvolvimento de sistemas especialistas.

\subsubsection{Interface com o usuário}

Nem todos os trabalhos que utilizaram ontologias apresentaram uma interface de fácil utilização. Premkumar et al. (2014) sugeriram que uma interface de ontologia amigável ao usuário deveria ser desenvolvida. Observa-se, portanto, que essa questão deve ser mais bem estudada para dominar as alternativas de integração de uma ferramenta visual com a ontologia. Uma alternativa surgiu do trabalho de Hiekata e Yamato (2014) que utilizaram Jena, uma API Java. Quanto a interação com o sistema, alguns autores, como Hernandez et al. (2012), desenvolveram uma interface onde o usuário pode responder questões ou fazer escolhas, o que se mostrou uma boa opção para operação de um sistema especialista.

\subsubsection{Outras incorporações para um sistema de DFM ou DFA}

Conforme realizado por Premkumar et al. (2014), integrar dados dos materiais em um sistema de DFM pode ser útil, uma vez que certas características da fabricação dependem do material utilizado. O estudo realizado por Ipek et al. (2013) contribuiu tecnicamente com esse propósito voltado para a seleção de material. Sapuan e Mansor (2014) sugeriram utilizar a Teoria da Solução de Problemas Inventivos (TRIZ) na abordagem de KBE. Já o trabalho de Hoque et al. (2013) inspira a considerar mais informações relativas às ferramentas de usinagem em um sistema voltado ao DFM. Observa-se ainda, a oportunidade de expandir o trabalho realizado por Hoque et al. (2013) voltado à usinagem para outros processos de fabricação.

\subsubsection{Integração}

Analisando o trabalho de Premkumar et al. (2014), observa-se que há maior facilidade em utilizar aplicações que forneçam saída nos formatos XML e XLS em conjunto com a ontologia. Portanto, aplicações com essa opção de formato de arquivo podem ser consideradas para uma integração com maior possibilidade de sucesso. 


\section{CONCLUSÃO}

O presente trabalho elucidou o estado da arte do emprego da Engenharia Baseada em conhecimento no projeto voltado à fabricação e montagem. A partir do processo ProKnow-C, realizaram-se etapas de seleção e triagem de artigos relacionados ao objeto de pesquisa, limitando-se aos últimos cinco anos, obtendo-se assim 22 trabalhos que compuseram o portfólio bibliográfico. A sequência se deu com a análise bibliométrica, que avaliou quantitativamente os trabalhos, e com a análise sistêmica, que permitiu conhecer os problemas, as soluções encontradas, e identificar as oportunidades de pesquisa geradas pelos trabalhos, se limitando nas três primeiras etapas do processo ProKnow-C.

Entre as oportunidades apresentadas, infere-se que entrevistas e a avaliação de situações semelhantes já ocorridas pode gerar uma solução futura para adquirir conhecimento na abordagem de DFMA por KBE. Além disso, ontologias poderiam ser utilizadas para estruturar o conhecimento obtido. Observou-se que há necessidade de delimitar a aplicação do $\mathrm{KBE}$, considerando que todos os trabalhos do portfólio foram específicos para uma finalidade: DFM, DFA ou outras. Conclui-se também que há oportunidade para explorar a aplicação de KBE para outros processos de fabricação não estudados, ou ainda, partir de trabalhos já realizados para avançar, podendo, por exemplo, considerar dados dos materiais e das ferramentas de fabricação em um sistema DFM ou utilizar o método da TRIZ para solução de problemas. Portanto, este trabalho poderá servir de apoio para o desenvolvimento futuro de uma solução ainda não identificada.

\section{REFERÊNCIAS}

ABDUL-GHAFOUR, S.; et al. Semantic interoperability of knowledge in feature-based CAD models, Computer-Aided Design, v56, p.45-57, 2014.

ANDERSON, F.; et al. Design for Manufacturing of Composite Structures for Commercial Aircraft - the Development of a DFM strategy at SAAB Aerostructures. Procedia CIRP, v17, p.362-367, 2014.

CANDIA, G.F.; et al. Knowledge based engineering system (KBES) for mechanic design activities. International Journal of Current Advanced Research, v5, n.6, p.984-991, 2016.

CHOWDARY, B.V.; MUTHINENI, S. Selection of a Flexible Machining Centre Through a Knowledge Based Expert System. Global Journal of Flexible Systems Management, v13, n.1, p.3-10, 2012.

DEMOLY, F; MATSOKIS, A.; KIRITSIS, D. A mereotopological product relationship description approach for assembly oriented design. Robotics and Computer-Integrated Manufacturing, v28,n.6, p.681-693, 2012.

ENSSLIN,L.; et al. ProKnow-C, knowledge development process-constructivist. Processo técnico com patente de registro pendente junto ao INPI. Brasil, v10,p.2015, 2010.

HERNANDEZ, N.V.; et al. Development of an expert system to aid engineers in the selection of design for environment methods and tools. Expert Systems with Applications, v39, n.10, p.9543-9553, 2012.

HIEKATA, K.; YAMATO, H. A study on process description method for DFM using ontology. CIRP Design Conference, p.210-215, 2014. 
HOQUE, A.S.M.; et al. Integrated manufacturing features and Design-for-manufacture guidelines for reducing product cost under CAD/CAM environment. Computers \& Industrial Engineering, v66, n.4, p.988-1003, 2013.

IPEK, M.; et. al. An expert system based material selection approach to manufacturing. Materials and Design, v47,p.331-340, 2013.

IWAYA, L.H.; ROSSO JUNIOR, R.S.U.; HOUNSELL, M.S. A Design for Assembly application with dynamic information retrieval from case database. IFAC Proceedings, v46, n.7, p.186-191, 2013.

JAKUBOWSKI, J.; PETERKA,J. Design for manufacturability in virtual environment using knowledge engineering. Management and Production Engineering Review, v5,n.1, p.3-10, 2014.

KRETSCHMER, R.; et al. Knowledge-based design for assembly in agile manufacturing by using Data Mining methods. Advanced Engineering Informatics, 2016.

KROGSTIEA, L.; ANDERSON, P. A Case Study on Reuse of Manufacturing Knowledge Comparing Defense Practices with Automotive \& Aerospace Practices. Procedia CIRP, v3, p.430-435, 2012.

LUETZENBERGER, J.; KLEIN, P.; THOBEN, K. Using knowledge based engineering to support the design of smart products. International Conference on Engineering Design, v6, p.63-72, 2013.

PREMKUMAR, V.; et al. A semantic knowledge management system for laminated composites. Advanced Engineering Informatics, v28,n.1, p.91-101, 2014.

RAMOS, L. Semantic Web for manufacturing, trends and open issues Toward a state of the art. Computers \& Industrial Engineering, v90, p.444-460, 2015.

ROZENFELD, H. et al. Gestão de Desenvolvimento de Produtos: uma referência para a melhoria do processo. São Paulo: Saraiva, 2006. 92 p.

SAPUAN, S.M.; MANSOR,M.R. Concurrent engineering approach in the development of composite products: A review. Materials and Design, v58, p.161-167, 2014.

WAHIDIN, L.S.; et al. A knowledge-based architecture framework of design for assemble system (DAEx). ARPN Journal of Engineering and Applied Sciences, v11, n.4, p.2505-2507, 2016.

ZHOU, Y.; SAITOU, K. Identification of manufacturability constraints through process simulation and data mining. ASME IDETC/CIE, v4, 2014. 